\title{
Netrin-1 induces the proliferation of gastric cancer cells via the ERK/MAPK signaling pathway and FAK activation
}

\author{
KAI YIN ${ }^{1,2^{*}}$, MENGYUAN SHANG ${ }^{3 *}$, SHENGCHUN DANG $^{1}$, LINJUN WANG $^{2}$, YIWEN XIA $^{2}$, \\ LEI CUI $^{1}$, XIN FAN $^{1}$, JIANGUO QU $^{1}$, JIXIANG CHEN ${ }^{1}$ and ZEKUAN XU ${ }^{2}$ \\ ${ }^{1}$ Department of General Surgery, Affiliated Hospital of Jiangsu University, Zhenjiang, Jiangsu 212001; \\ ${ }^{2}$ Department of General Surgery, The First Affiliated Hospital of Nanjing Medical University, Nanjing, Jiangsu 210000; \\ ${ }^{3}$ Department of Ultrasound Diagnosis, Affiliated Hospital of Jiangsu University, Zhenjiang, Jiangsu 212001, P.R. China
}

Received December 1, 2017; Accepted July 27, 2018

DOI: $10.3892 / o r .2018 .6614$

\begin{abstract}
Netrin-1 (NTN1) has been demonstrated to promote tumorigenesis in multiple types of cancer; however, its role in the growth of gastric cancer (GC) cells has not been described in detail. In the present study, the data suggested that NTN1 knockdown significantly decreased the proliferation of GC cells, whereas NTN1 overexpression had an opposing effect. Furthermore, the use of focal adhesion kinase (FAK) inhibitor decreased the proliferation of GC cells. It was also revealed that NTN1 markedly induced the phosphorylation of FAK, extracellular signal-regulated kinase (ERK) and c-Jun N-terminal kinase (JNK), but did not induce the phosphorylation of P38. In addition, the expression of ERK and JNK was markedly inhibited by treatment with FAK inhibitor. Xenograft analysis using GC cells revealed that NTN1 overexpression promoted tumor growth. Furthermore, the expression of NTN1 in samples collected from nude mice was downregulated in the NTN1 knockdown group and upregulated in the NTN1 overexpression group compared with the control short hairpin RNA group. These results suggest that NTN1-induced GC cell proliferation is mediated by activating ERK/MAPK signaling cascades via the distinct activation of FAK.
\end{abstract}

Correspondence to: Professor Zekuan Xu, Department of General Surgery, The First Affiliated Hospital of Nanjing Medical University, 300 Guangzhou Road, Nanjing, Jiangsu 210000, P.R. China E-mail: xuzekuan@njmu.edu.cn

Professor Jixiang Chen, Department of General Surgery, Affiliated Hospital of Jiangsu University, 438 Jiefang Road, Zhenjiang, Jiangsu 212001, P.R. China

E-mail: cyok9@163.com

${ }^{*}$ Contributed equally

Key words: Netrin-1, gastric cancer, proliferation, mitogen-activated protein kinase, extracellular signal-regulated kinase, focal adhesion kinase

\section{Introduction}

Gastric cancer (GC), the fourth most common type of cancer, has been demonstrated to be the third leading cause of cancer-associated mortality in males and the fifth leading cause of cancer-associated mortality in females worldwide (1). In order to develop an effective treatment for GC, the molecular mechanisms of its development and progression must be elucidated. However, in the past decade, limited progress has occurred in studying the progression of GC.

Netrin-1 (NTN1) is a laminin-like protein that was initially identified as one of the axonal guidance molecules in neuronal cell development (2). NTN1 has several dependent receptors, including uncoordinated-5-homolog (UNC5H), which consists of $\mathrm{UNC} 5 \mathrm{H} 1, \mathrm{UNC} 5 \mathrm{H} 2, \mathrm{UNC} 5 \mathrm{H} 3$ and $\mathrm{UNC} 5 \mathrm{H} 4$, deleted in colorectal cancer (DCC), neogenin and Down syndrome cell adhesion molecule. Previous studies have demonstrated that NTN1 mRNA and protein expression is associated with numerous types of cancer, including colorectal $(3,4)$, hepatic (5), neuroblastoma (6), breast (7), pancreatic $(8,9)$, prostate (10) and non-small cell lung cancer (11). In addition, NTN1 has been demonstrated to regulate cancer cell proliferation, migration, invasion and apoptosis $(5,8,9,12,13)$. Our previous study revealed that NTN1 promoted GC cells growth via its receptor neogenin (14); however, the downstream signaling pathway remains to be elucidated.

Numerous studies have reported that focal adhesion kinase (FAK) may be one of the primary downstream effector molecules of NTN1 in neural system development $(15,16)$. Previous findings have indicated that NTN1 induces mesenchymal stem cell motility via the distinct activation of FAK (17). In addition, a number of studies reported the proliferation-promoting effect of NTN1 in renal tubular epithelial cells was induced via activation of the extracellular signal-regulated kinase (ERK)/mitogen-activated protein kinase (MAPK) signaling pathway $(18,19)$. However, the coordination of signal transduction cascades downstream of NTN1 via FAK remains unclear in GC development. Thus, investigating the mechanisms of the NTN1/FAK-mediated signaling pathway in the regulation of GC cell proliferation is of importance.

In the present study, the role of NTN1 in promoting the growth of GC cells and its associated signaling pathways 
was investigated. It was demonstrated that NTN1 significantly promoted GC cells proliferation in vitro and in vivo. Furthermore, the present results suggest that NTN1-induced GC cells proliferation was mediated by the ERK/MAPK signaling pathway via activation of FAK.

\section{Materials and methods}

Cell culture and reagents. The human GC cell lines (SGC7901 and MGC803) were obtained from the Cell Bank of the Chinese Academy of Medical Science (Shanghai, China). All cell lines were cultured in RPMI-1640 (Gibco; Thermo Fisher Scientific, Inc., Waltham, MA, USA) containing $10 \%$ fetal bovine serum (Invitrogen; Thermo Fisher Scientific, Inc.), penicillin (100 U/ml) and streptomycin $(100 \mathrm{mg} / \mathrm{ml})$ at $37^{\circ} \mathrm{C}$ in an atmosphere containing $5 \% \mathrm{CO}_{2}$. MEK inhibitor U0126 and FAK inhibitor PF562271 were purchased from Selleck Chemicals (Houston, TX, USA). GC cells were pretreated with PF562271 $(10 \mu \mathrm{M})$ and U0126 $(10 \mu \mathrm{M})$ for $24 \mathrm{~h}$ to inhibit MEK and FAK expression.

RNA interference and lentivirus transfection. The polybrene lentivirus transfection reagent was purchased from Shanghai GenePharma Co., Ltd. (Shanghai, China). The shRNAs targeting NTN1 (5'-CATGGAGCTCTACAAGCTT-3') and scramble shRNA (5'-GTTCTCCGAACGTGTCACGT-3') were synthesized and ligated into a retroviral vector (Shanghai GenePharma Co., Ltd.). DNA fragments corresponding to NTN1, which were amplified from human genomic DNA, were cloned into the lentivirus for NTN1 overexpression (Shanghai GenePharma Co., Ltd.). For lentivirus transfection, $2 \times 10^{5}$ SGC7901 and MGC803 cells were plated into 6-well plates. After $24 \mathrm{~h}$, cells were washed with PBS 3 times, and then cultured with 2 ml RPMI-1640, $100 \mu 1$ NTN1 overexpression or knockdown lentivirus $\left(1 \times 10^{8}\right.$ transducing units $\left./ \mathrm{ml}\right)$ and polybrene $(5 \mu \mathrm{g} / \mathrm{ml})$. Retrovirus packaging and transfection were conducted according to the manufacturer's protocol.

Cell proliferation assay. Cell proliferation was determined using a CCK-8 assay (Dojindo Molecular Technologies, Inc., Kumamoto, Japan). GC cells transfected with lentivirus were seeded into 96-well plates at a density of $1 \times 10^{3}$ cells/well in triplicate and incubated with RPMI-1640 supplemented with $10 \%$ FBS for 5 days. CCK-8 solution $(10 \mu \mathrm{l})$ was added to each plate at various time points $(24,48,72,96$ and $120 \mathrm{~h})$ and cells were incubated for $2 \mathrm{~h}$ at $37^{\circ} \mathrm{C}$. The absorbance was measured according to the manufacturer's protocol.

Cell cycle assay using flow cytometry (FCM). Transfected GC cells were digested with trypsin and centrifuged at $1,000 \mathrm{xg}$ for $5 \mathrm{~min}$ at room temperature. Subsequently, the cells were washed with PBS 3 times and fixed in $75 \%$ ethanol at $4^{\circ} \mathrm{C}$ overnight. Prior to FCM detection, cells were washed twice with PBS, incubated with RNAse at room temperature for $15 \mathrm{~min}$, stained with $500 \mu \mathrm{l}$ propidium iodide staining solution for $15 \mathrm{~min}$ at room temperature. Data was obtained using a FACScan flow cytometer with BD Cell Quest Pro 5.0 software (both from BD Biosciences, Franklin Lakes, NJ, USA).

Reverse transcription-quantitative polymerase chain reaction (RT-qPCR). Total RNA was isolated from tumor xenograft tissues using TRIzol reagent (Invitrogen; Thermo Fisher Scientific, Inc.) following the manufacturer's protocol. cDNA was produced from RNA by RT using a PrimeScript RT reagent kit (Takara Biotechnology Co., Ltd., Dalian, China). The RT temperature protocol used was as follows: $15 \mathrm{~min}$ at $37^{\circ} \mathrm{C}$; reverse transcriptase inactivation for $5 \mathrm{sec}$ at $85^{\circ} \mathrm{C}$; followed by storage at $4^{\circ} \mathrm{C}$. The primer sequences were as follows: $\beta$-actin forward, 5'-AGATCCTGACCGAGCGTG GC-3' and reverse, 5'-CCAGGGAGGAAGAGGATGCG-3'; NTN1 forward, 5'-AAGCAGGGCACAAGTCGTAT-3' and reverse, 5'-TGCTCTTGTCTGCCACGATG-3'. qPCR was performed using SYBR Green Real-time PCR Master mix (Roche Diagnostics GmbH, Mannheim, Germany). Briefly, the qPCR was performed on an Applied Biosystems 7500 Fast Real-Time PCR system (Applied Biosystems; Thermo Fisher Scientific, Inc.) with the following thermal profile: Hot-start DNA polymerase activation to $95^{\circ} \mathrm{C}$ for $10 \mathrm{~min}$; 40 cycles of $95^{\circ} \mathrm{C}$ for $15 \mathrm{sec}$ and $60^{\circ} \mathrm{C}$ for $1 \mathrm{~min}$; followed by melt curve analysis at $95^{\circ} \mathrm{C}$ for $15 \mathrm{sec}, 60^{\circ} \mathrm{C}$ for $1 \mathrm{~min}$ and $95^{\circ} \mathrm{C}$ for $15 \mathrm{sec}$. Relative expression of NTN1 mRNA in tissues was analyzed according to the $2^{-\Delta \Delta \mathrm{Cq}}$ method (20). $\beta$-actin used as an internal control gene.

Western blot analysis. SGC7901 and MGC803 cells were harvested for protein extraction according to standard procedures. Cell extracts were prepared for western blotting using a Total Protein Extraction kit (cat. no. KGP2100; Nanjing KeyGen Biotech Co., Ltd., Nanjing, China). Subsequently, a BCA protein assay was used for protein quantification. A total of $40 \mu \mathrm{g}$ of protein extracts from each group were resolved using $10 \%$ SDS-PAGE and transferred to polyvinylidene fluoride membranes. Following blocking with 5\% skimmed milk at room temperature for $2 \mathrm{~h}$, the membranes were incubated with specific primary antibodies in dilution buffer at $4^{\circ} \mathrm{C}$ overnight. The following primary antibodies were used: NTN1 (cat. no. ab126729; anti-rabbit; 1:200 dilution; Abcam, Cambridge, UK), GAPDH (cat. no. 51332; anti-mouse), phosphorylated (p)-FAK (cat. no. 3284; anti-rabbit), FAK (cat. no. 13009; anti-rabbit), p-c-Jun N-terminal kinase (JNK) (cat. no. 4668; anti-rabbit), JNK (cat. no. 9252; anti-rabbit), p-ERK (cat. no. 4376; anti-rabbit), ERK (cat. no. 4695; anti-rabbit), p-P38 (cat. no. 4631; anti-rabbit) and P38 (cat. no. 8690; anti-rabbit) (all 1:1,000 dilution; all from Cell Signaling Technology, Inc., Danvers, MA, USA). GAPDH was used as an internal control. Following this, the membranes were washed 3 times with TBS-Tween-20 and incubated with horseradish peroxidase (HRP)-conjugated anti-mouse (cat. no., GAM007; 1:1,000 dilution) or anti-rabbit IgG (cat.no. GAB007; 1:1,000 dilution) (both from Hangzhou Multi Sciences Biotech Co., Ltd., Hangzhou, China) at room temperature for $2 \mathrm{~h}$. Bands were visualized using ECL Plus (EMD Millipore, Billerica, MA, USA) with a FluorChem E enhanced chemiluminescence detection system (ProteinSimple, San Jose, CA, USA).

Tumor xenograft with human GC cells in nude mice. A total of 15, 4-week-old male nude BALB/c mice (weight range, 13-15 g) were purchased from the Department of Laboratory Animal Centre of Yangzhou University (Yangzhou, China) and divided into 3 groups at random. All mice were raised 
under pathogen-free conditions, including at room temperature $\left(21-26^{\circ} \mathrm{C}\right)$ and $12 \mathrm{~h} \mathrm{light} /$ dark cycle. The mice received $150 \mathrm{ml}$ water and $5 \mathrm{~g}$ food per $100 \mathrm{~g}$ body weight per day. SGC7901 and MGC803 cells (NTN1 knockdown, control and NTN1 overexpression) were implanted into the right flank of the nude mice by subcutaneous injection $\left(2 \times 10^{6}\right.$ cells in $100 \mu \mathrm{l}$ of PBS) to promote tumor formation. Bidimensional tumor diameters were measured using a slide caliper every 7 days. Nude mice were euthanized after 4 weeks. Xenograft tumor volume was calculated using the following formula: $(a b)^{2} \times(0.5)^{2}$, whereby a represents the length of the tumor and $b$ represents the width diameter of the tumor (21). The present study was approved by the Jiangsu University Animal Ethics Committee (Zhenjiang, China).

Immunohistochemical staining (IHC). Xenograft tumor samples were fixed in $4 \%$ formalin at room temperature overnight and embedded in paraffin. Then, the sections were washed in PBS 3 times and blocked in 5\% bovine serum albumin (Servicebio Technology Co., Ltd., Wuhan, China) for $30 \mathrm{~min}$ at room temperature. The $4-\mu \mathrm{m}$ slices were incubated with ki-67 antibody (cat. no. ab15580; anti-rabbit; 1:100 dilution; Abcam) at $4^{\circ} \mathrm{C}$ overnight and washed with PBS 3 times. Following incubation with HRP-polymer-conjugated secondary antibody (cat. no. ab6721; goat anti-rabbit; 1:1,000 dilution; Abcam) at room temperature for $1 \mathrm{~h}$, the slices were dyed with diaminobenzidine solution for $3 \mathrm{~min}$ at room temperature and then stained with $0.2 \%$ hematoxylin at room temperature for $3 \mathrm{~min}$. The sections were observed with an inverted microscope (original magnification, x100; NIKON ECLIPSE TI-SR; Nikon Corporation, Tokyo, Japan). Ki-67 expression levels were evaluated according to the percentage of positively stained tumor cells and cell staining intensity. The staining intensity was graded as follows: 0 (no staining); 1 (weak); 2 (moderate); and 3 (strong). The following scores were used to describe the overall proportion of $\mathrm{Ki}-67$ positive cells: 0 (negative); $1(<30 \%) ; 2(30-60 \%)$; and $3(>60 \%)$. The two scores were multiplied, with scores $\geq 4$ being defined as high expression, and scores $<4$ as low expression.

Statistical analysis. Statistical analysis was performed using SPSS software (version 22; IBM, Corp., Armonk, NY, USA). Data are presented as the mean \pm standard error of the mean. Differences between 2 groups were evaluated using paired student t-tests. Data from $>2$ groups were compared with two-way analysis of variance followed by a least-significant difference post hoc test. $\mathrm{P}<0.05$ was considered to indicate a statistically significant difference.

\section{Results}

NTN1 enhances GC cells proliferation in vitro. To investigate the role of NTN1 on GC cells proliferation, MGC803 and SGC7901 cells were transfected with lentivirus to overexpress or knock down NTN1. As shown in Fig. 1A, NTN1 protein expression was markedly decreased following silencing and increased following overexpression, indicating that transfection was successful. CCK- 8 results revealed that NTN1 silencing significantly decreased the proliferation of GC cells compared with the control group. By contrast,
NTN1 overexpression notably enhanced cell proliferation in MGC803 and SGC7901 cells (Fig. 1B and C). To further explore the mechanism responsible for the NTN1-induced GC growth, the cell cycle distribution in each group was investigated using FCM. Similarly, NTN1 knockdown in MGC803 and SGC7901 cells significantly increased the percentage of cells in $G_{0} / G_{1}$ phase, whereas the opposite was observed in NTN1-overexpressed cells (Fig. 1D and E). Taken together, these results suggest that NTN1 overexpression promoted GC cells proliferation, while NTN1 knockdown induced cell cycle arrest at the $G_{0} / G_{1}$ phases.

Enhanced GC cell proliferation induced by NTN1 is FAK dependent. The effect of NTN1 on the phosphorylation of FAK, which is considered to be essential in netrin signaling pathways $(16,22)$, was examined. The results indicated that PF562271, a FAK inhibitor, markedly suppressed FAK phosphorylation in MGC803 and SGC7901 cell lines (Fig. 2A). The results also demonstrated that the proliferation of GC cells pretreated with PF562271 was significantly inhibited (Fig. 2B and C). In order to demonstrate that NTN1-induced GC cells growth was mediated by FAK, FCM was used to assess the distribution of GC cells in the cell cycle following NTN1 knockdown and overexpression. As shown in Fig. 2D and E, PF56227 significantly increased the percentage of cells in the $\mathrm{G}_{0} / \mathrm{G}_{1}$ phases in MGC803 and SGC7901 cells.

Effects of NTN1 on the ERK/MAPK signaling pathway. The ERK/MAPK signaling pathway is the primary downstream pathway of FAK (22). Whether ERK/MAPK signal cascades participate in NTN1-enhanced GC cell proliferation was investigated. It was demonstrated that FAK, ERK and JNK phosphorylation were reduced in MGC803 and SGC7901 cells following NTN1 knockdown compared with the negative control group. By contrast, increased phosphorylation of FAK, ERK and JNK was observed in the NTN1 overexpression group, while no changes were detected in the total protein levels (Fig. 3A). However, another downstream target of MAPK signaling pathway, P38, was not significantly altered in GC cells following NTN1 knockdown and overexpression. To verify the role of NTN1 in signaling pathways mediated by FAK, MGC803 and SGC7901 cells with NTN1 overexpression were treated with PF56227 for $24 \mathrm{~h}$. The results revealed that PF56227 significantly inhibited NTN1-induced phosphorylation of ERK and JNK (Fig. 3B).

Effects of ERK on GC cells proliferation ability in vitro. The effect of ERK on GC cell proliferation was analyzed. ERK phosphorylation was suppressed in MGC803 and SGC7901 cell lines following treatment with MEK inhibitor U0126 (Fig. 4A). The results demonstrated that pretreatment with the MEK inhibitor (U0126) also downregulated GC cell proliferation (Fig. 4B and C) and increased the percentage of cells in the $G_{0} / G_{1}$ phases (Fig. 4D and E). Taken together, these results demonstrated that the ERK/MAPK signaling pathway may be involved in enhancing GC cell proliferation and that NTN1 exerted this effect through the activation of FAK.

NTN1 promotes tumor growth in nude mice inoculated with GC cells. In order to investigate the effect of NTN1 on tumor 


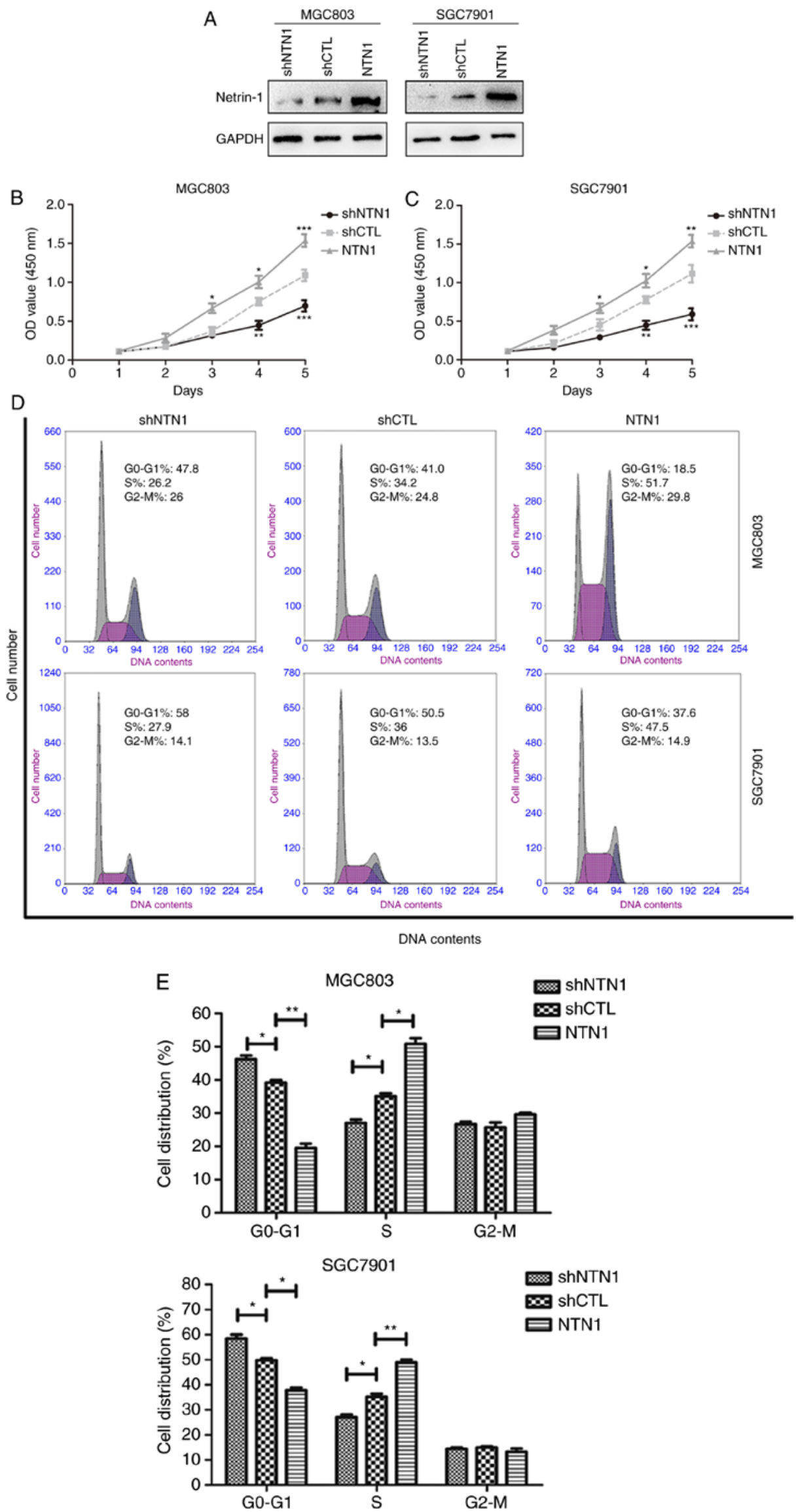

Figure 1. Effect of NTN1 on gastric cancer cell proliferation. (A) NTN1 was efficiently decreased following transfection with NTN1 knockdown lentivirus in MGC803 and SGC7901 cells. NTN1 was increased following transfection with NTN1 overexpression lentivirus. NTN1 expression was examined by western blot analysis following transfection for $48 \mathrm{~h}$. The Cell Counting Kit-8 assay indicated that NTN1 knockdown suppressed the proliferation abilities of (B) MGC803 and (C) SGC7901 cells. (D) The effect of NTN1 on the GC cell cycle distribution was analyzed using flow cytometry and (E) quantified. "P<0.05, ${ }^{* *} \mathrm{P}<0.01$ and ${ }^{* * *} \mathrm{P}<0.001$. NTN1, Netrin-1; sh, shRNA; CTL, control. 
A $\quad$ MGC803 $\quad$ SGC7901

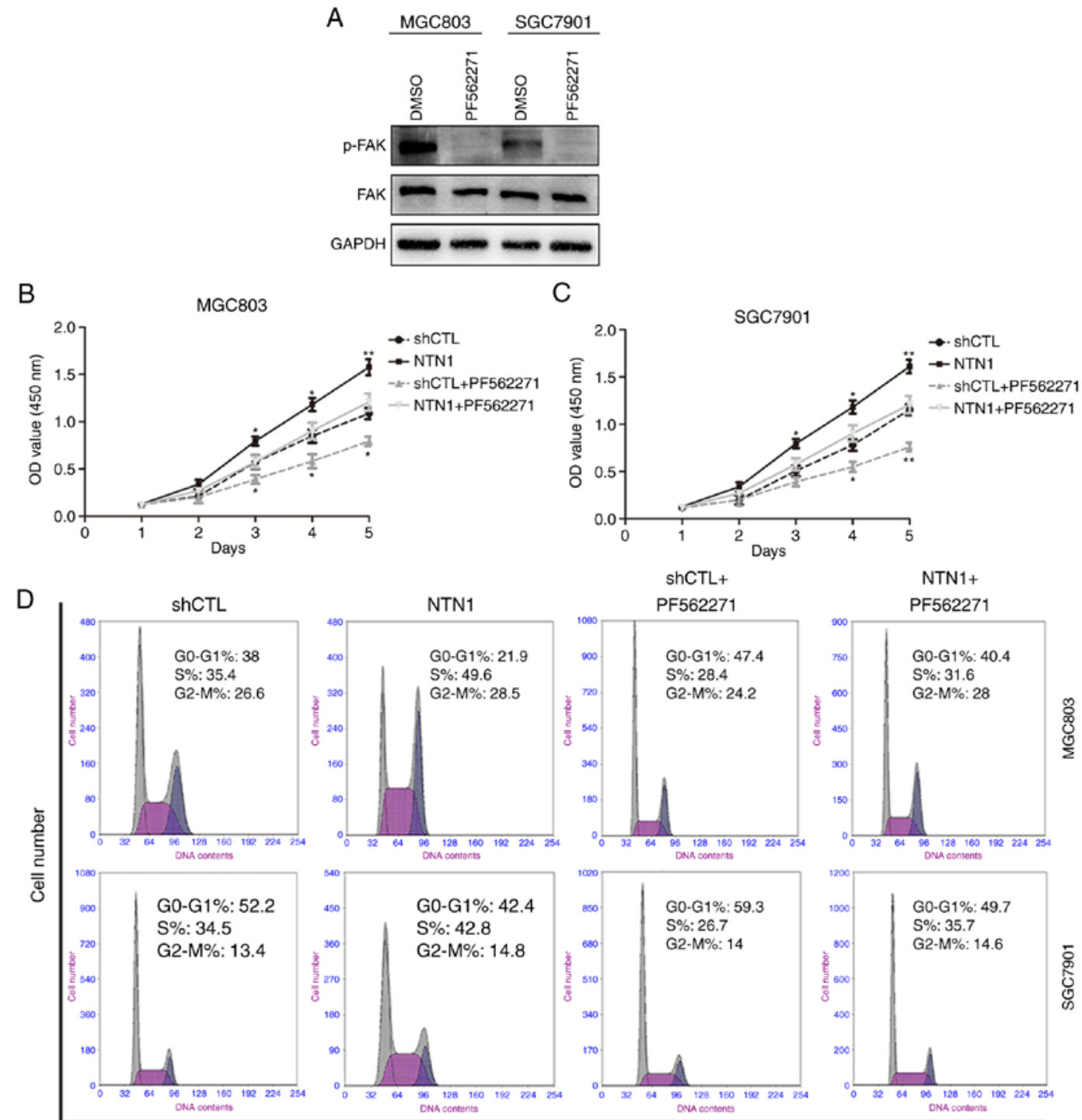

DNA contents

E
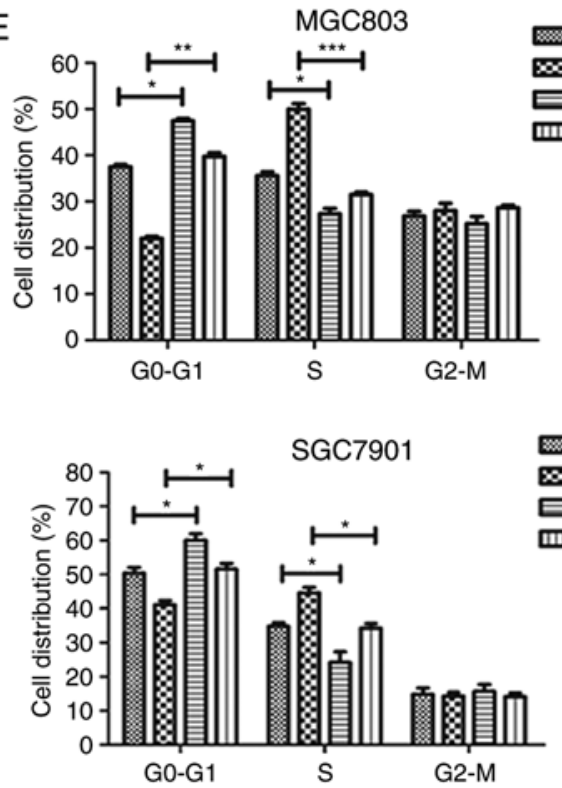

Figure 2. Effects of NTN1 on the activation of FAK in vitro. (A) Western blot analysis was used to analyze the expression of FAK in MGC803 and SGC7901 cells pretreated with FAK inhibitor (PF562271). The Cell Counting Kit-8 assay indicated that the FAK inhibitor (PF562271) decreased the proliferation ability of (B) MGC803 and (C) SGC7901 cells transfected with lentivirus. A cell cycle assay was (D) performed and (E) quantified, which indicated that FAK inhibitor (PF562271) increased the percentage of cells in $\mathrm{G}_{0} / \mathrm{G}_{1}$ phase in MGC803 and SGC7901 cells. ${ }^{*} \mathrm{P}<0.05,{ }^{* * *} \mathrm{P}<0.01$ and ${ }^{* * * *} \mathrm{P}<0.001$. FAK, focal adhesion kinase; NTN1, Netrin-1; sh, shRNA; CTL, control; p, phosphorylated. 

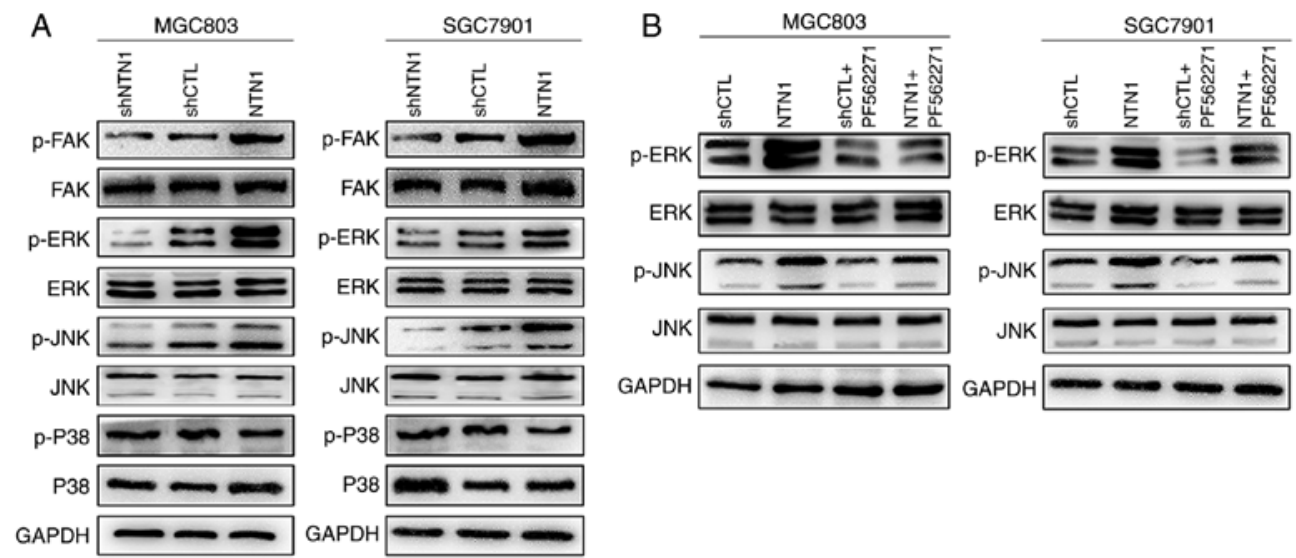

Figure 3. Effects of NTN1 on the ERK/MAPK signaling pathway are dependent on FAK. (A) MGC803 and SGC7901 cells were transfected with NTN1 knockdown or overexpression lentivirus, and the expression levels of p-FAK/FAK, p-ERK/ERK, p-JNK/JNK and p-P38/P38 were analyzed using western blot analysis. (B) Western blot analysis revealed that pretreatment with FAK inhibitor (PF562271) altered the expression levels of p-ERK/ERK and p-JNK/JNK. FAK, focal adhesion kinase; NTN1, Netrin-1; sh, shRNA; CTL, control; p, phosphorylated; JNK, -Jun N-terminal kinase; ERK, extracellular signal-regulated kinase.

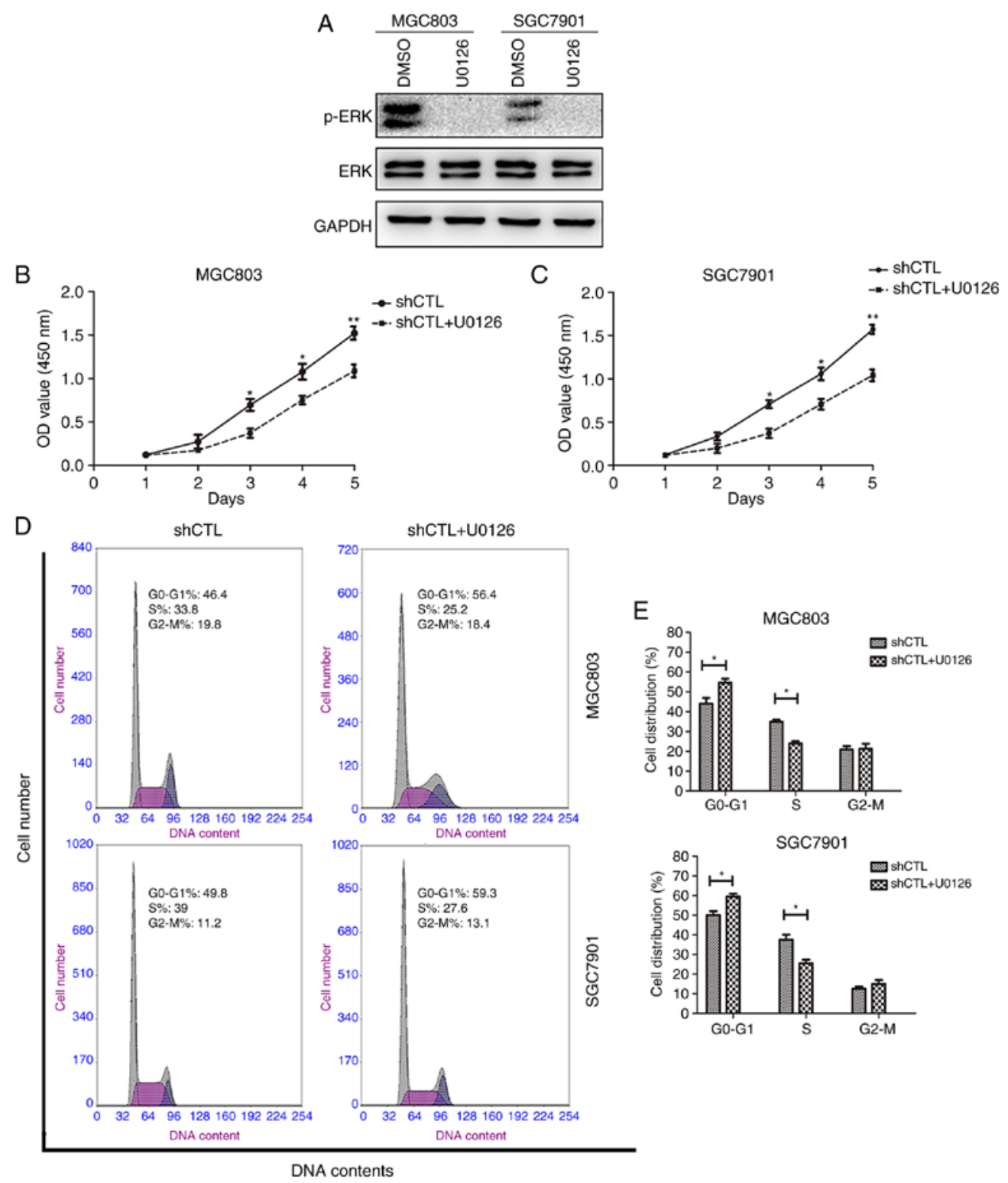

Figure 4. Effect of ERK on gastric cancer cell proliferation in vitro. (A) Western blot analysis was used to analyze the expression of ERK in MGC803 and SGC7901 cells pretreated with MEK inhibitor (U0126). The proliferation abilities of (B) MGC803 and (C) SGC7901 cells pretreated with MEK inhibitor (U0126) were determined using a Cell Counting Kit-8 assay. Cell cycle analysis was (D) performed and (E) quantified, which indicated that the MEK inhibitor (U0126) increased the percentage of cells in $\mathrm{G}_{0} / \mathrm{G}_{1}$ phase in MGC803 and SGC7901 cells. " $\mathrm{P}<0.05$ and ${ }^{* *} \mathrm{P}<0.01$. sh, shRNA; CTL, control; p, phosphorylated; ERK, extracellular signal-regulated kinase. 
A

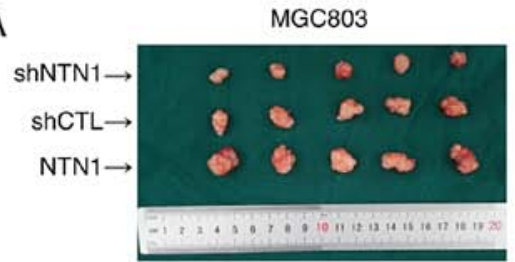

D

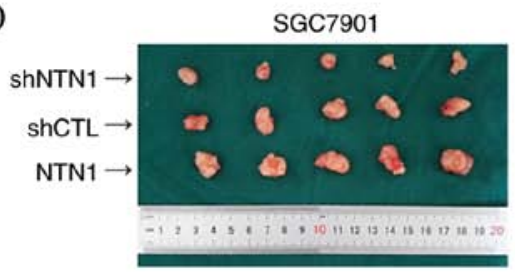

B

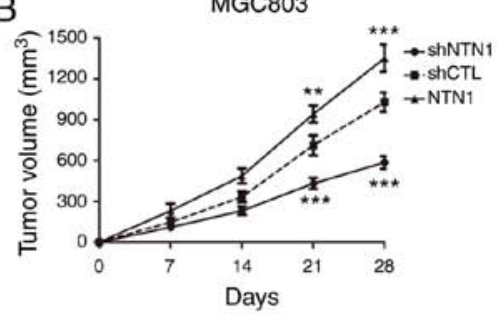

E

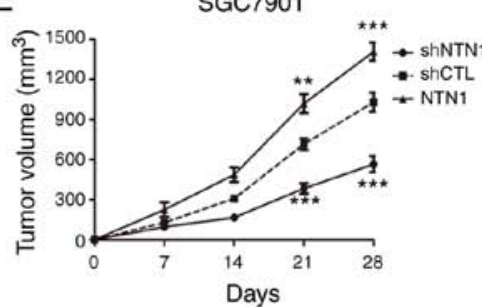

C

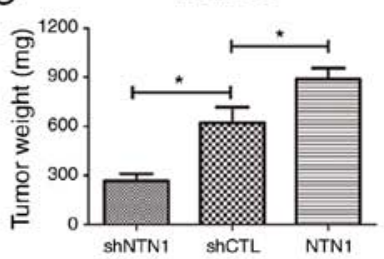

F
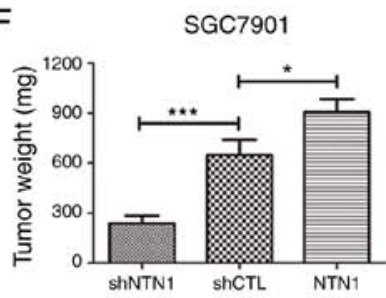

G

$\mathrm{H}$

\section{SGC7901}

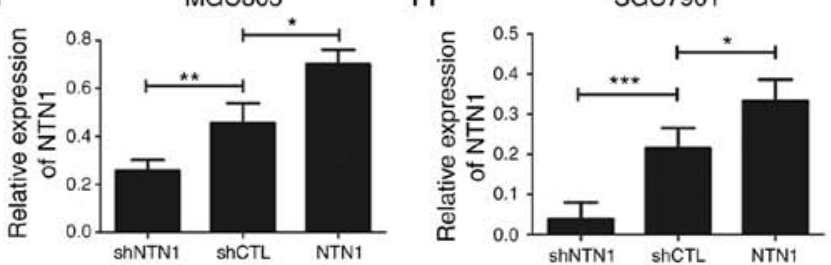

I ShNTN1

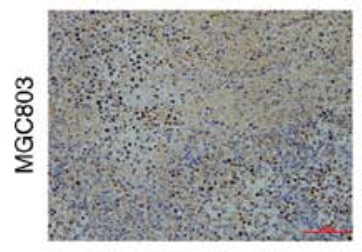

J

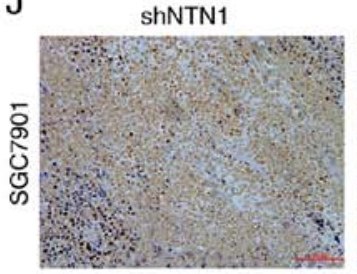

shCTL

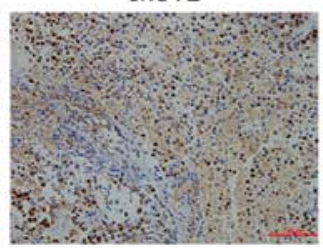

ShCTL

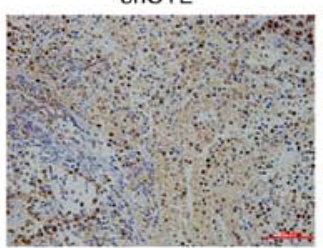

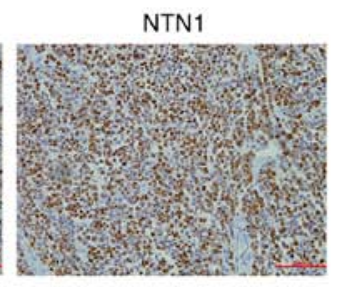

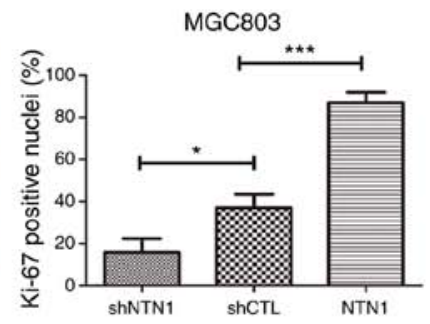

NTN1

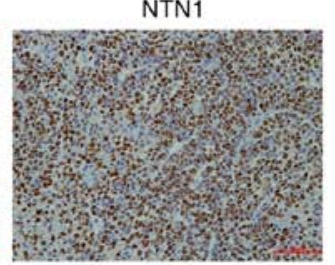

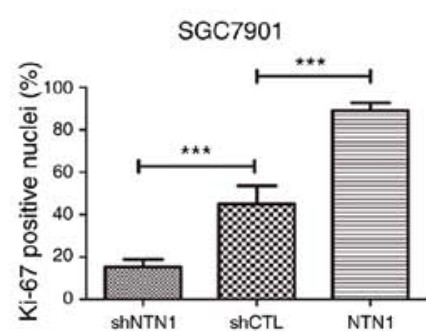

Figure 5. In vivo analysis of NTN1 in the regulation of gastric cancer cell growth. (A) Following transfection with NTN1 knockdown or overexpression lentivirus, MGC803 cells were injected into the right flank of nude mice. Mice were sacrificed after 4 weeks and tumors were obtained from nude mice. Representative images of isolated tumors in the NTN1 knockdown group (first row), control group (second row) and NTN1 overexpression group (third row). Tumor (B) volume and (C) weight were examined at 5 different time points following injection with MGC803 cells with NTN1 knockdown or overexpression. (D) SGC7901 cells were injected into the right flank of nude mice. Representative images of isolated tumors in the NTN1 knockdown group (first row), control group (second row) and NTN1 overexpression group (third row). Tumor (E) volume and (F) weight were examined at 5 different time points following injection with SGC7901 cells with NTN1 knockdown or overexpression. The expression of NTN1 in tissues collected from nude mice that were injected with (G) MGC803 and (H) SGC7901 cells was examined by reverse transcription-quantitative polymerase chain reaction. Ki-67 IHC staining was used to determine the effects of NTN1 expression on the cell proliferation abilities in the samples collected from nude mice injected with (I) MGC803 and (J) SGC7901 cells. Original magnification, $\mathrm{x} 100$; Scale bar, $100 \mu \mathrm{m}$. ${ }^{*} \mathrm{P}<0.05,{ }^{* *} \mathrm{P}<0.01$ and ${ }^{* * * *} \mathrm{P}<0.001$. NTN1, Netrin-1; sh, shRNA; CTL, control.

growth in nude mice, GC cells (NTN1 knockdown, control and NTN1 overexpression) were injected into the right flank of nude mice to form ectopic tumors. As shown in Fig. 5A-F, the tumor volume and weight in the NTN1 knockdown group were decreased compared with the control group. Conversely, the tumor volume and weight in the NTN1 overexpression group were increased compared with the control group. Furthermore, the relative expression of NTN1 in samples collected from nude mice was measured using RT-qPCR. As shown in Fig. 5G and H, the expression of NTN1 was downregulated in the NTN1 knockdown group, while it was upregulated in the NTN1 overexpression group. Ki-67 IHC analysis was performed to assess GC cell proliferation in vivo. The positive nuclei rate of $\mathrm{Ki}-67$ was lower in the NTN1 knockdown group and higher in NTN1 overexpression group (Fig. 5I and J). Taken together, these results indicate 
that proliferation ability of GC cells was impaired following NTN1 knockdown, which was consistent with the experimental results in vitro.

\section{Discussion}

The proto-oncogene NTN1 has been reported to promote cancer cell proliferation during tumor development $(7,8,23)$. In the present study, the in vivo and in vitro results identified that the nerve-derived molecule NTN1 has the ability to stimulate GC cell proliferation, suggesting that NTN1 has an oncogenic effect. Furthermore, it was revealed that NTN1 induces the distinct activation of FAK, subsequently leading to the induction of the ERK/MAPK signaling pathway.

FAK is a cytoplasmic protein tyrosine kinase, which has been demonstrated to be involved in cancer cell progression, including cell proliferation and migration (24). Previous studies have reported that FAK may be one of the primary downstream effectors of NTN1, functioning through the receptors DCC and neogenin $(15,16)$. Furthermore, it has been reported that NTN1-induced cell proliferation and migration is mediated by the activation of FAK $(17,25)$. The results of the present study suggest that FAK phosphorylation was decreased following NTN1 knockdown in GC cells, whereas NTN1 overexpression had the opposite effect. GC cell proliferation was significantly inhibited following pretreatment with FAK inhibitor, which is consistent with previous results $(26,27)$. These results indicate that dysfunction of FAK signaling pathway may be an important mechanism involved in GC cell proliferation.

The ERK/MAPK signaling pathway has been demonstrated to serve important roles in the proliferation of various cells (28). In addition, the results of the present study revealed that ERK and JNK phosphorylation levels were increased following NTN1 overexpression, which has been confirmed to be a downstream effect of NTN1 in other studies $(5,18)$. Notably, the mechanism by which NTN1 induces renal proximal tubular epithelial cells proliferation has been proposed as stimulation of the UNC5B, which triggers the activation of the ERK/MAPK signaling pathway (18). The results in the current study indicated that NTN1 knockdown decreased the phosphorylation of ERK and JNK, while P38 phosphorylation was unchanged. Furthermore, the present results suggest that the addition of the MEK inhibitor suppressed the proliferation ability of NTN1 in GC cells. However, it has been reported that NTN1 stimulates p38/MAPK, but not ERK/MAPK signaling pathways in Schwann cells (29). Notably, it was also reported that NTN1 suppressed the growth of pancreatic cancer via inhibition of the ERK/MAPK signaling pathway through its receptor UNC5B (30). In addition, NTN1 has been demonstrated to induce the inhibition of the ERK/MAPK signaling pathway during lung branching morphogenesis (31). These findings suggest that NTN1 possibly has bidirectional effects on the ERK/MAPK signaling pathway depending on the receptor types or ligand structure.

In conclusion, the present study demonstrated that the NTN1-induced promotion of GC cell proliferation was mediated via activation of the FAK/ERK/MAPK signaling pathway in vivo and in vitro. Considering the role of NTN1 on GC progression, the therapeutic potential of NTN1 in GC requires further investigation and evaluation.

\section{Acknowledgements}

Not applicable.

\section{Funding}

The present study was supported by the Jiangsu Province Fund for 'Six talent summits' high-level talent (grant no. 2016-WSN-007) and Zhenjiang Key Research and Development Plan (grant nos. SH2015066 and SH2017023).

\section{Availability of data and materials}

All data generated or analyzed during this study are available upon reasonable request from the corresponding author.

\section{Authors' contributions}

ZX and JC conceived and designed the study. KY, MS, YX and SD performed the experiments and acquired data. LW, JQ, LC and XF analyzed the data. KY drafted and edited the manuscript. All authors have given final approval of the version to be published.

\section{Ethics approval and consent to participate}

The present study was approved by the Jiangsu University Animal Ethics Committee.

\section{Patient consent for publication}

Not applicable.

\section{Competing interests}

The authors declare that they have no competing interest.

\section{References}

1. Torre LA, Bray F, Siegel RL, Ferlay J, Lortet-Tieulent J and Jemal A: Global cancer statistics, 2012. CA Cancer J Clin 65: 87-108, 2015.

2. Lai Wing Sun K, Correia JP and Kennedy TE: Netrins: Versatile extracellular cues with diverse functions. Development 138: 2153-2169, 2011.

3. Mazelin L, Bernet A, Bonod-Bidaud C, Pays L, Arnaud S, Gespach C, Bredesen DE, Scoazec JY and Mehlen P: Netrin-1 controls colorectal tumorigenesis by regulating apoptosis. Nature 431: 80-84, 2004.

4. Ko SY, Blatch GL and Dass CR: Netrin-1 as a potential target for metastatic cancer: Focus on colorectal cancer. Cancer Metastasis Rev 33: 101-113, 2014.

5. Han P, Fu Y, Liu J, Wang Y, He J, Gong J, Li M, Tan Q, Li D, Luo Y, et al: Netrin-1 promotes cell migration and invasion by down-regulation of BVES expression in human hepatocellular carcinoma. Am J Cancer Res 5: 1396-1409, 2015.

6. Delloye-Bourgeois C, Fitamant J, Paradisi A, Cappellen D, Douc-Rasy S, Raquin MA, Stupack D, Nakagawara A, Rousseau R, Combaret V, et al: Netrin-1 acts as a survival factor for aggressive neuroblastoma. J Exp Med 206: 833-847, 2009.

7. Fitamant J, Guenebeaud C, Coissieux MM, Guix C, Treilleux I, Scoazec JY, Bachelot T, Bernet A and Mehlen P: Netrin-1 expression confers a selective advantage for tumor cell survival in metastatic breast cancer. Proc Natl Acad Sci USA 105: 4850-4855, 2008.

8. Dumartin L, Quemener C, Laklai H, Herbert J, Bicknell R, Bousquet C, Pyronnet S, Castronovo V, Schilling MK, Bikfalvi A and Hagedorn M: Netrin-1 mediates early events in pancreatic adenocarcinoma progression, acting on tumor and endothelial cells. Gastroenterology 138: 1595-1606, 1606 e1591-1598, 2010. 
9. Huang Q, Hua HW, Jiang F, Liu DH and Ding G: Netrin-1 promoted pancreatic cancer cell proliferation by upregulation of Mdm2. Tumour Biol 35: 9927-9934, 2014.

10. Kong CZ, Liu J, Liu L, Zhang Z and Guo KF: Interactional expression of netrin-1 and its dependence receptor UNC5B in prostate carcinoma. Tumour Biol 34: 2765-2772, 2013.

11. Delloye-Bourgeois C, Brambilla E, Coissieux MM, Guenebeaud C, Pedeux R, Firlej V, Cabon F, Brambilla C, Mehlen $\mathrm{P}$ and Bernet A: Interference with netrin-1 and tumor cell death in non-small cell lung cancer. J Natl Cancer Inst 101: 237-247, 2009.

12. Akino T, Han X, Nakayama H, McNeish B, Zurakowski D, Mammoto A, Klagsbrun M and Smith E: Netrin-1 promotes medulloblastoma cell invasiveness and angiogenesis, and demonstrates elevated expression in tumor tissue and urine of patients with pediatric medulloblastoma. Cancer Res 74: 3716-3726, 2014

13. Shimizu A, Nakayama H, Wang P, König C, Akino T, Sandlund J, Coma S, Italiano JE Jr, Mammoto A, Bielenberg DR, et al: Netrin-1 promotes glioblastoma cell invasiveness and angiogenesis by multiple pathways including activation of RhoA cathepsin B, and cAMP-response element-binding protein. J Biol Chem 288: 2210-2222, 2013 .

14. Yin K, Wang LJ, Zhang X, He Z, Xia Y, Xu J, Wei S, Li B, Li Z, Sun G, et al: Netrin-1 promotes gastric cancer cell proliferation and invasion via the receptor neogenin through PI3K/AKT signaling pathway. Oncotarget 8: 51177-51189, 2017.

15. Liu G, Beggs H, Jurgensen C, Park HT, Tang H, Gorski J Jones KR, Reichardt LF, Wu J and Rao Y: Netrin requires focal adhesion kinase and Src family kinases for axon outgrowth and attraction. Nat Neurosci 7: 1222-1232, 2004.

16. Moore SW, Zhang X, Lynch CD and Sheetz MP: Netrin-1 attracts axons through FAK-dependent mechanotransduction. J Neurosci 32: 11574-11585, 2012

17. Lee SJ, Jung YH, Oh SY, Yong MS, Ryu JM and Han HJ: Netrin-1 induces MMP-12-dependent E-cadherin degradation via the distinct activation of PKC $\alpha$ and FAK/Fyn in promoting mesenchymal stem cell motility. Stem Cells Dev 23: 1870-1882, 2014.

18. Wang W, Reeves WB and Ramesh G: Netrin-1 increases proliferation and migration of renal proximal tubular epithelial cells via the UNC5B receptor. Am J Physiol Renal Physiol 296 : F723-F729, 2009.

19. Mohamed R, Jayakumar C, Ranganathan PV, Ganapathy V and Ramesh G: Kidney proximal tubular epithelial-specific overexpression of netrin-1 suppresses inflammation and albuminuria through suppression of COX-2-mediated PGE2 production in streptozotocin-induced diabetic mice. Am J Pathol 181: 1991-2002, 2012.
20. Livak KJ and Schmittgen TD: Analysis of relative gene expression data using real-time quantitative PCR and the $2^{-\Delta \Delta C \mathrm{~T}}$ method. Methods 25: 402-408, 2001.

21. Naito S, von Eschenbach AC, Giavazzi R and Fidler IJ: Growth and metastasis of tumor cells isolated from a human renal cell carcinoma implanted into different organs of nude mice. Cancer Res 46: 4109-4115, 1986.

22. Li W, Lee J, Vikis HG, Lee SH, Liu G, Aurandt J, Shen TL, Fearon ER, Guan JL, Han M, et al: Activation of FAK and Src are receptor-proximal events required for netrin signaling. Nat Neurosci 7: 1213-1221, 2004

23. Rodrigues S, De Wever O, Bruyneel E, Rooney RJ and Gespach C: Opposing roles of netrin- 1 and the dependence receptor DCC in cancer cell invasion, tumor growth and metastasis. Oncogene 26: 5615-5625, 2007.

24. Ying X, Huang A, Xing Y, Lan L, Yi Z and He P: Lycorine inhibits breast cancer growth and metastasis via inducing apoptosis and blocking Src/FAK-involved pathway. Sci China Life Sci 60: 417-428, 2017

25. Yang X, Li S, Zhong J, Zhang W, Hua X, Li B and Sun H: CD151 mediates netrin-1-induced angiogenesis through the Src-FAK-Paxillin pathway. J Cell Mol Med 21: 72-80, 2017.

26. Cheng Z, Liu F, Zhang H, Li X, Li Y, Li J, Liu F, Cao Y, Cao L and Li F: miR-135a inhibits tumor metastasis and angiogenesis by targeting FAK pathway. Oncotarget 8: 31153-31168, 2017.

27. Zang M, Zhang Y, Zhang B, Hu L, Li J, Fan Z, Wang H, Su L, Zhu Z, Li C, et al: CEACAM6 promotes tumor angiogenesis and vasculogenic mimicry in gastric cancer via FAK signaling. Biochim Biophys Acta 1852: 1020-1028, 2015.

28. Razidlo GL, Kortum RL, Haferbier JL and Lewis RE: Phosphorylation regulates KSR1 stability, ERK activation, and cell proliferation. J Biol Chem 279: 47808-47814, 2004.

29. Lv J, Sun X, Ma J, Ma X, Zhang Y, Li F, Li Y and Zhao Z: Netrin-1 induces the migration of Schwann cells via p38 MAPK and PI3K-Akt signaling pathway mediated by the UNC5B receptor. Biochem Biophys Res Commun 464: 263-268, 2015.

30. An XZ, Zhao ZG, Luo YX, Zhang R, Tang XQ, Hao D, Zhao X, Lv X and Liu D: Netrin-1 suppresses the MEK/ERK pathway and ITGB4 in pancreatic cancer. Oncotarget 7: 24719-24733, 2016.

31. Liu Y, Stein E, Oliver T, Li Y, Brunken WJ, Koch M, Tessier-Lavigne M and Hogan BL: Novel role for Netrins in regulating epithelial behavior during lung branching morphogenesis. Curr Biol 14: 897-905, 2004. 